\title{
Dynamic monitoring and evaluation of ecological restoration in mining area based on GNSS+RS technology
}

\author{
Qiang Wang* \\ Shengli Oilfield Technology Inspection Center, SINOPEC , 257000 , Dongying, Shandong, \\ China
}

\begin{abstract}
The continuous development of mineral resources is increasingly damaging the ecological environment, so it is of great significance to ecological restoration and dynamic monitoring of the mining area. In this paper, dynamic monitoring and evaluation method of ecological restoration in the mining area are proposed, which integrates GNSS + RS (Global Navigation Satellite System + Remote Sensing) technology. According to the Precipitable Water Vapor (PWV) retrieved by GNSS and NDVI (Normalized Vegetation Index) can monitor the ecological environment and introduce machine learning to improve the accuracy of the model. The dynamic assessment of ecological restoration was carried out by using temperature, rainfall, NPP (Net Primary Productivity), NDVI and PWV. The results show that: (1) the modeling effect of machine learning is better than that of the least square regression. (2) The comprehensive ecological evaluation index proposed can better reflect the ecological situation of the mining area. Therefore, the environmental monitoring and assessment of mining area based on GNNS + RS technology proposed in this paper have important reference significance.
\end{abstract}

Keywords: GNSS, RS, Ecological restoration, Comprehensive assessment.

\section{Introduction}

As non-renewable resources, mineral resources are the material guarantee for the survival and development of human society [1]. Exploitation and utilization of mineral resources is an important supporting means to meet China's economic development in the energy field, but mining will inevitably cause damage to the ecological and geological environment of mining areas and aggravate the deterioration of the ecological environment [2]. The ecological restoration and reconstruction after mining is an important guarantee for the sustainable development of mining area, but how to dynamically monitor and evaluate the ecological environment in mining area, determine the degree of restoration and reconstruction and give the specific quantitative index is a difficult problem that needs to be solved urgently at home and abroad [3].

\footnotetext{
*Corresponding author: chengyayu12345@163.com
} 
Mining will cause serious damage to the ecological environment, mainly in the aspects of reduced land use, landscape destruction, mountain destruction, vegetation destruction, glaciers, geological hazards and increased atmospheric dust concentration [4]. Therefore, accurate dynamic monitoring and accurate assessment of ecological restoration in mining area is of great significance to further implement ecological restoration planning, prevent and avoid secondary damage to ecological environment caused by unreasonable planning, and promote sustainable development of mining area [5]. For the ecological restoration after mining, vegetation restoration is the key point of ecological restoration and the important foundation of ecological restoration. Vegetation can purify the suspended particles in the atmosphere, reduce the dust phenomenon, improve the regional atmospheric environment, and achieve the purpose of ecological environment restoration.

In this paper, the dynamic monitoring and evaluation methods of ecological restoration in mining areas in Shanxi Province are studied, and the dynamic monitoring and evaluation are realized by studying the parameters of PWV, NPP, temperature, rainfall and NDVI in different urban areas in Shanxi Province. The regression model of PWV and NDVI of GNSS inversion was established by the least square method. The ecological environment was monitored by the GNSS data indirectly through the regression model, and the temporal and spatial characteristics of NPP, temperature, rainfall and NDVI were analyzed. In addition, BP (Back Propagation) neural network and SVM (Support Vector Machine) support vector machine are used to model and analyze the multi-ecological factors and NDVI, finally, the trend of vegetation coverage and comprehensive ecological index in Shanxi Province and cities is analyzed.

\section{Data sources and research methods}

\subsection{Data introduction}

\subsubsection{Acquisition of atmospheric precipitable water based on GNSS}

Atmospheric precipitable water is the total amount of water vapor in a unit gas column from the surface to the top of the atmosphere, which can represent the potential of precipitation and is an important basis for evaluating air water resources [6]. The atmospheric precipitable water can be retrieved through the foundation GNSS mainly consists of two steps: firstly, ZHD (Zenith Hydrostatic Delay)[7] is calculated by the Saastamoinen model, ZWD (Zenith Wet Delay) is obtained by ZTD (Tm), and ZWD is converted into PWV by the conversion parameters obtained by the weighted average atmospheric temperature $\mathrm{Tm}$.

\subsubsection{Vegetation net primary productivity}

Vegetation net primary productivity (NPP) refers to the amount of organic matter accumulated by green plants through photosynthesis per unit area and time. The net primary productivity of vegetation is an important index to evaluate the coordination of ecosystem structure and function and its interaction with the environment, and is a direct index to express the energy and material quantity of ecosystem [8]. The vegetation net primary productivity index used in this paper is derived from NASA (National Aeronautics and Space Administration, NASA) annual NPP data at MOD 17A3 spatial resolution of $1 \mathrm{~km}$ from 2000 to 2015, obtained by https://ladsweb.modaps.eosdis.nasa.gov/search/. 


\subsubsection{Normalized vegetation index}

Normalized Vegetation Index(NDVI) is an important index to reflect vegetation growth . It is of great significance for ecological protection,returning farmland to forest and disaste prevention to explore its temporal and spatial variation characteristics[9]. The GIMMS NDVI3g data of Shanxi Province is from the NASA GIMMS AVHRR Global NDVI3g $1 / 12$ degree data set of Clark Laboratory in the United States. According to the https://ecocast.arc.nasa.gov/data/pub/gimms/3g.v1, the spatial and temporal resolution is 15 days and $8 \mathrm{~km}$. The data is preprocessed and calculated by the maximum synthesis method to obtain the NDVI data of Shanxi Province from 1981 to 2015.

\subsubsection{Normalized water body index}

Normalized water body index (NDWI) is an improved method based on NDWI. According to the reflection intensity analysis of plants and water in visible and near-infrared bands, a water body index extraction method is proposed to minimize vegetation information and enhance water body information in images[10]. In this paper, the remote sensing image data of NDWI is calculated from the geospatial data cloud (http://www.gscloud.cn/). According to the satellite digital products, the NDWI data of Shanxi Province in 2000,2005,2010 and 2015 are calculated.

\subsection{Research methods}

\subsubsection{Pearson correlation coefficient}

In this paper, the temperature, rainfall and vegetation index from 2000 to 2016 are analyzed by using linear regression analysis. The linear regression model is obtained by using the least square method, and the variation trend of each factor is judged by the slope. Pearson correlation coefficient is used to calculate the correlation between factors according to formula (1). Pearson correlation coefficient is used to measure the linear correlation between two variables, which is an index reflecting the close relationship between variables [11].

$$
r=\frac{\sum\left(X_{i}-\bar{X}\right)\left(Y_{i}-\bar{Y}\right)}{\sqrt{\sum\left(X_{i}-\bar{X}\right)^{2} \sum\left(Y_{i}-\bar{Y}\right)^{2}}}
$$

where, $r$ is dimensionless normalized covariance, and the value of $r$ is between \pm 1 . The greater the absolute value of $r$, the stronger the correlation between the two variables. $r>0$ indicates positive correlation between variables, $r<0$ indicates negative correlation between two variables.

\subsubsection{Multi-factor model construction based on machine learning}

This paper uses BP neural network and SVM support vector machine to model the ecological factors of Shanxi Province, and analyzes the deep relationship of ecological assessment factors. BP neural network is a multi-layer feedforward neural network trained according to the error back propagation algorithm, which is the most extensive neural network at present [12]. The input and output of each neuron in the implicit layer can be calculated by formula (2), and the global error E can be calculated by formula (3). When the error meets the requirements, the algorithm is ended, or the next sample is selected for training and learning. 


$$
\begin{cases}h i_{h}(\mathrm{k})=\sum_{i=1}^{n} w_{i h} x_{i}(\mathrm{k})-\mathrm{b}_{n} & \\ h o_{h}(\mathrm{k})=f\left(h i_{h}(\mathrm{k})\right) & \mathrm{h}=1,2, \cdots, \mathrm{p} \\ y i_{o}(\mathrm{k})=\sum_{h=1}^{p} w_{h o} h o_{h}(\mathrm{k})-\mathrm{b}_{o} & \mathrm{o}=1,2, \cdots, \mathrm{q} \\ y o_{o}(\mathrm{k})=f\left(y i_{o}(\mathrm{k})\right) & \\ E=\frac{1}{2} \sum_{k=1}^{m} \sum_{o=1}^{q}\left(d_{o}(k)-y_{o}(k)\right)^{2}\end{cases}
$$

wherein, $h_{h}(k)$ is the input vector of the hidden layer of the kth input sample at the hth nerve cell; $\mathrm{ho}_{\mathrm{h}}(\mathrm{k})$ is the output vector of the hidden layer of the kth input sample at the hth nerve cell;

$\mathrm{yi}_{\mathrm{o}}(\mathrm{k})$ is the input vector of the output layer of the kth input sample at the oth nerve cell; $\mathrm{yo}_{\mathrm{o}}(\mathrm{k})$ is the output vector of the output layer of the kth input sample at the othnerve cell; $\mathrm{w}_{\mathrm{ih}}$ is the connection weight between the output layer and the middle layer; $\mathrm{w}_{\mathrm{ho}}$ is the connection weight of the hidden layer and the output layer; $b_{h}$ is the threshold of each neuron of the hidden layer; $b_{o}$ is the threshold of each neuron of the output layer; $f$ is the activation function;

Sample data number $\mathrm{k}=1,2, \ldots . . \mathrm{m}$.

Support Vector Machine (SVM) is a class of generalized linear classifier which classifies the data into two parts according to supervised learning. Its decision boundary is the maximum margin hyperplane for learning sample solution [13]. According to the best effect between model complexity and prediction ability of specific training sample, it can be divided into support vector classification and support vector regression. This paper mainly carries out model construction based on support vector regression algorithm, and the SVM regression coefficient is determined by Lagrangian multiplier.

\subsubsection{System vulnerability level}

The quantitative assessment of ecosystem vulnerability can provide a corresponding decision-making basis for the sustainable development of ecosystems, the reduction of adverse impacts of external stress on ecosystems and the comprehensive remediation of degraded ecosystems [14]. Modelled on the IPCC definition of system vulnerability as a function of sensitivity and adaptability, the relationship between system vulnerability, system sensitivity and system adaptability is given [15] :

$$
\mathrm{V}=\mathrm{S}-\mathrm{A}
$$

where, $\mathrm{V}$ is system vulnerability, $\mathrm{S}$ is system sensitivity, i.e. the degree of influence of the system on the change of external factors, and A is system adaptability or adaptability. Based on the analysis of sensitivity and adaptability, K-means clustering algorithm was used to classify the vulnerability of ecosystem into five grades: non-fragile, mild-fragile, moderate-fragile, severe-fragile and extreme-fragile, which were used as indicators to evaluate the improvement or deterioration of ecosystem.

\section{Multi-factor and NDVI dynamic monitoring model construction}

\subsection{NDVI and multi-factor spatio-temporal characteristic analysis}

Since the urban mean evaluation factors cannot represent the development trend of each region, to understand the rate of change of each city, Figure 1 shows the interannual change 
trend of NDVI, PWV and NPP in each region. Figure a shows that there is no obvious interannual variation trend of NDVI in each region. The growth rate in the west of Lvliang City is higher than that in the east, the growth rate in the south of Taiyuan City is lower than that in the north, the growth rate in the middle and the north of Jinzhong City is faster, the NDVI growth rate in Jincheng City and Yuncheng City is generally lower. Observation Figure b shows that PWV change rate law is obvious, the decline rate of PWV from the north to the south gradually becomes larger, and the growth rate in Yuncheng City and Jincheng City is the most prominent; Figure c shows that the change rate of NPP does not have certain regularity, except for some regions in the middle of Shanxi Province and some regions in the south of Shanxi Province, and the growth rate in the west is higher than that in the east.
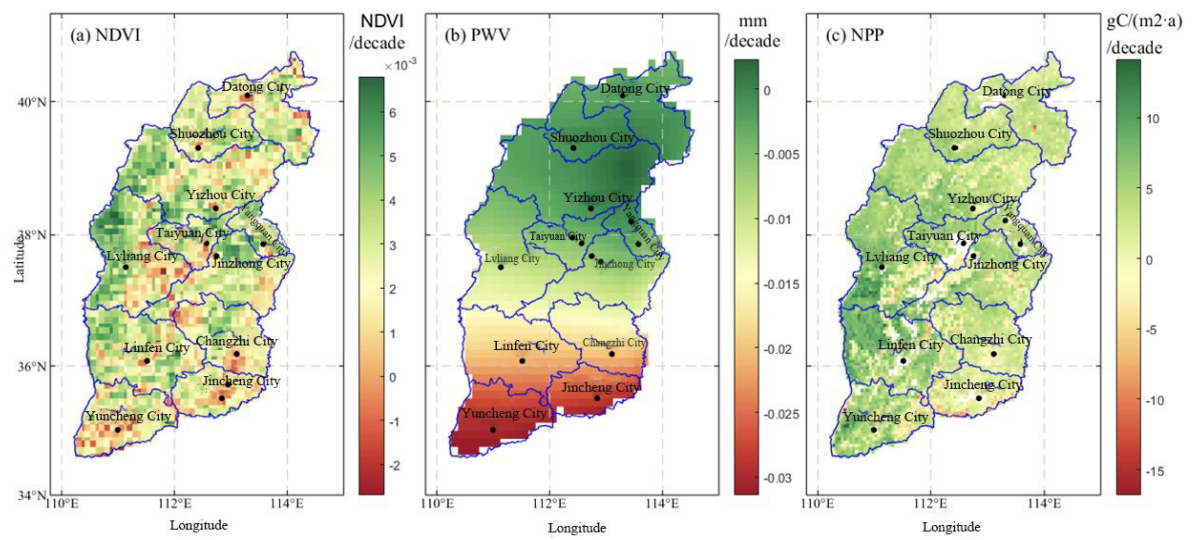

Fig. 1. Inter annual trend of NDVI, PWV and NPP grid outlets in Shanxi Province.

As the grid data of temperature and rainfall is incomplete, the analysis is completed based on each meteorological station, and the results are shown in Figure 2. Fig. a shows that the temperature increase rate in northern Shanxi except Datong is better than that in southern Shanxi, in which the temperature increase rate of the gas-image station in Xinzhou is the highest, and the temperature increase rate of the meteorological stations in Yuncheng is the lowest in southern Shanxi; Fig. b shows that the rainfall change rate in Shanxi is relatively regular, in which the northern and central regions of Shanxi are dominated by the growth trend, while the south, especially in Yuncheng, has the obvious decreasing trend.
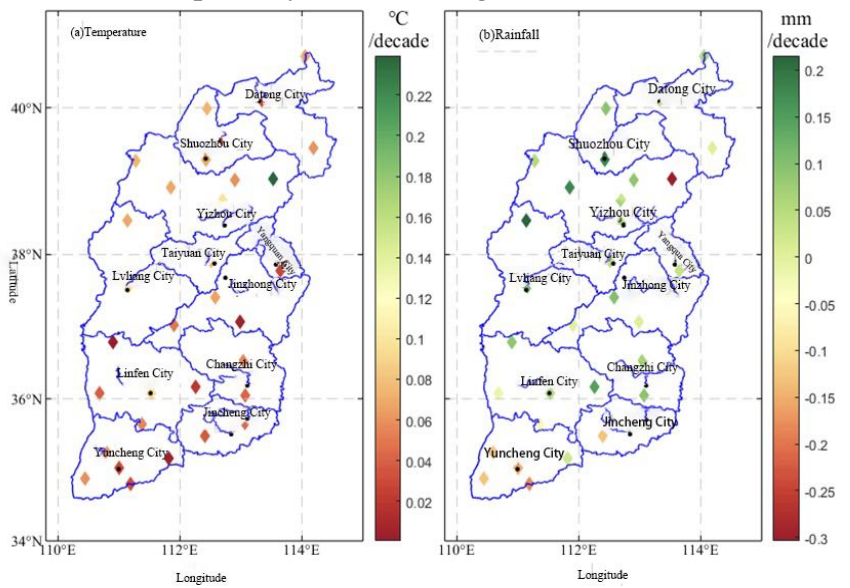

Fig. 2. Annual variation trend of temperature and rainfall at meteorological stations in Shanxi Province. 


\subsection{Modeling based on least squares regression}

The NDVI is mainly affected by climate change and human activities. In consideration of other influencing factors, PWV factor is introduced and its correlation coefficient with NDVI is calculated. Linear regression equation is established by selecting relevant PWV data combined with annual average NDVI. Fig.3 shows the regression coefficient distribution of NDVI and PWV and temperature in each city of Shanxi Province, and it is found that the regression coefficient of NDVI and PWV is greater than 0 in Fig. a, among which the regression coefficient in the north of Shanxi Province, such as Xinzhou City, Shuozhou City and southeast of Datong City, is relatively large, and the regression coefficient of PWV and NDVI is relatively small in Shanxi Province is relatively small. According to Fig. b, the regression coefficient of NDVI and temperature in each city of Shanxi Province is greater than 0, indicating that the temperature and NDVI show the same change trend. Among them, the regression coefficients in Xinzhou, Changzhi and Jinzhong meteorological stations are relatively large, while those in Lvliang, Taiyuan, Shuozhou and northern Datong are relatively small.
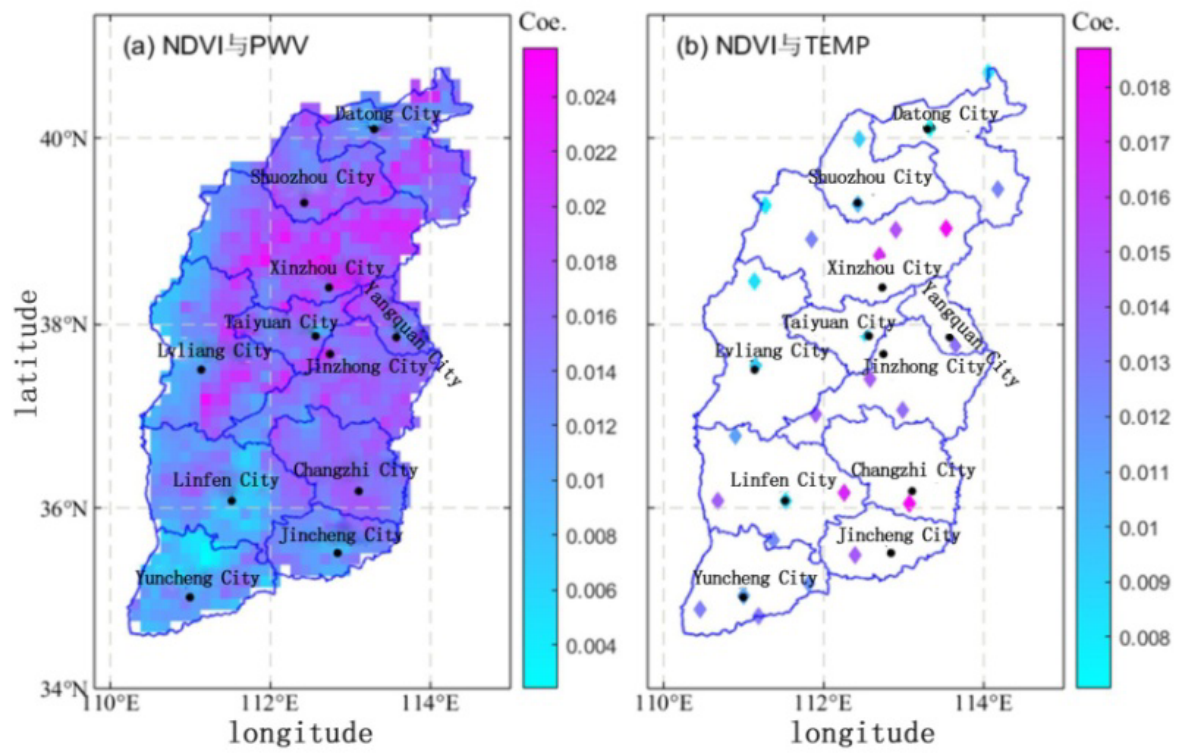

Fig. 3. Regression coefficient of NDVI, PWV and temperature in Shanxi Province.

\subsection{Modeling based on machine learning}

In order to further explore the deep relationship between NDVI and temperature, rainfall and PWV and other ecological assessment factors, SVM support vector machine (SVM) and BP neural network (BPNNN) were used to construct ecological model for NDVI and temperature rainfall, NDVI and temperature rainfall and PWV respectively. The results are shown in Figure 4. SVM support vector machine (SVM) is a kind of small sample machine learning method with relatively perfect theoretical basis, which can solve the high-dimensional problem [16]. Therefore, the temperature and rainfall model based on SVM support vector machine has certain accuracy, and the model accuracy is improved correspondingly after PWV evaluation factor is introduced. With the ability of learning to approximate arbitrary nonlinear mapping, the neural network can be applied to the identification and prediction of nonlinear systems, and can not be limited by nonlinear models, so that the BP neural network can realize the mapping function from input to 
output, which is suitable for solving complex internal problems, and has self-learning and adaptive ability [17]. Compared with SVM support vector mechanism model, BP neural network model is more accurate than PWV model, and the model is more accurate than temperature and rainfall model.
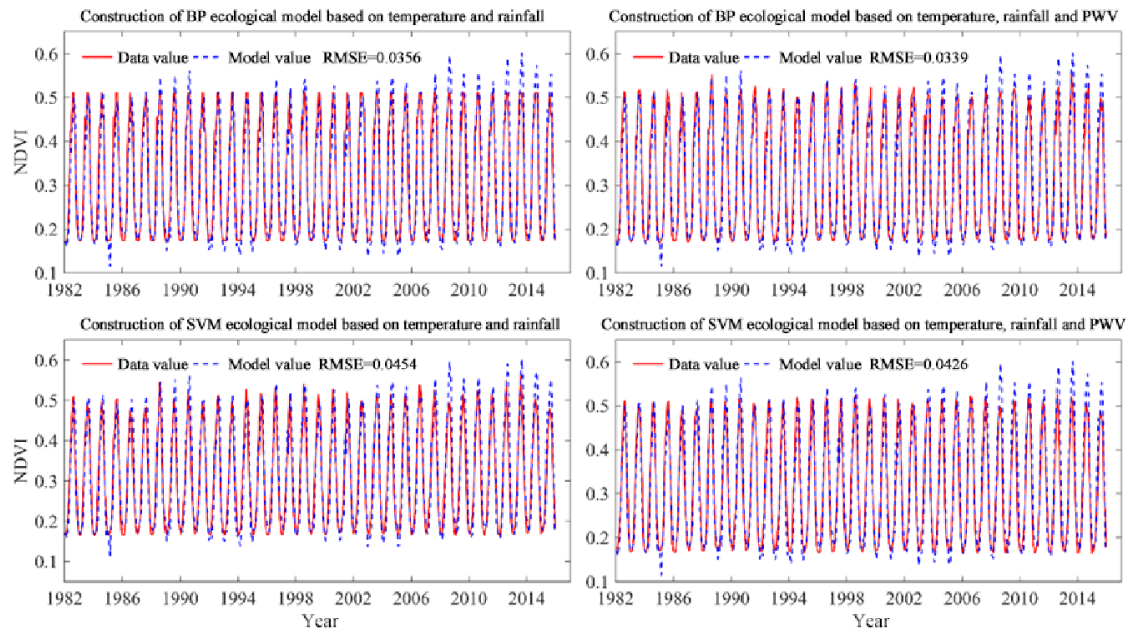

Fig. 4. Model construction based on machine learning.

\section{Evaluation assessment and comprehensive index}

\subsection{Single factor ecological evaluation}

\subsubsection{Water body change}

In this paper, Xiaruyue Reservoir, Dongyulin Reservoir and Huliu River and Hotan Village Reservoir of Guangling County are selected as the main water body extraction areas for statistical water body area change, as shown in Figure 5. The resolution of image data in different periods is the same $(30 \mathrm{~m})$, but the sensor performance is different, which results in the difference of extracted pixel area. The overall variation trend of water body area shows a decreasing trend, and the vegetation area shows a decreasing trend. Due to the limitation of image data quality and NDWI algorithm, it is impossible to judge the increase or decrease of specific water body area by the numerical value of statistical area, but the ecological environment monitoring in the study area is a good evaluation factor. 

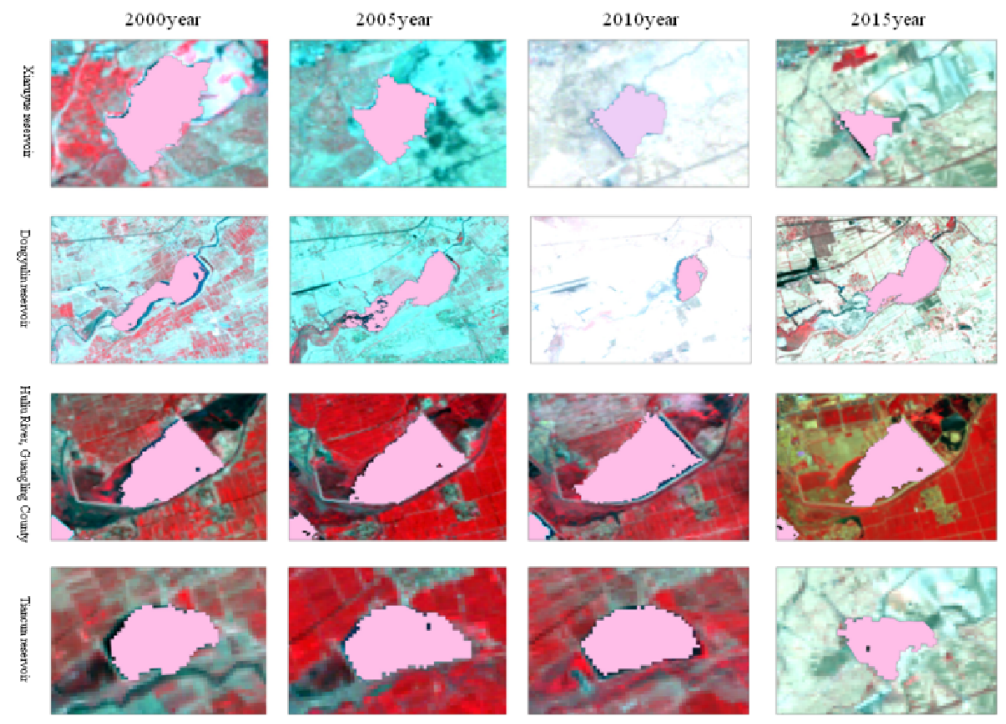

Fig. 5. Multi period water extraction map of each region.

\subsubsection{Interannual variation of vegetation coverage}

Figure 6 shows the annual variation trend of vegetation coverage in each city in Shanxi Province. The vegetation coverage of Datong City, Jincheng City, Yangquan City, Changzhi City and Taiyuan City in Shanxi Province shows a downward trend, which may be related to the change of land use.The vegetation coverage of Shuozhou City, Yizhou City, Luliang City, Jinzhong City, Linfen City and Yuncheng City shows an upward trend, especially the hilly-gully region between Luliang Mountain and Yellow River is the key ecological protection area in ChinaThe vegetation coverage of this area is in an upward trend due to afforestation. As a whole, the vegetation coverage of Shanxi Province is decreasing. This may be directly related to coal mining in Shanxi Province.
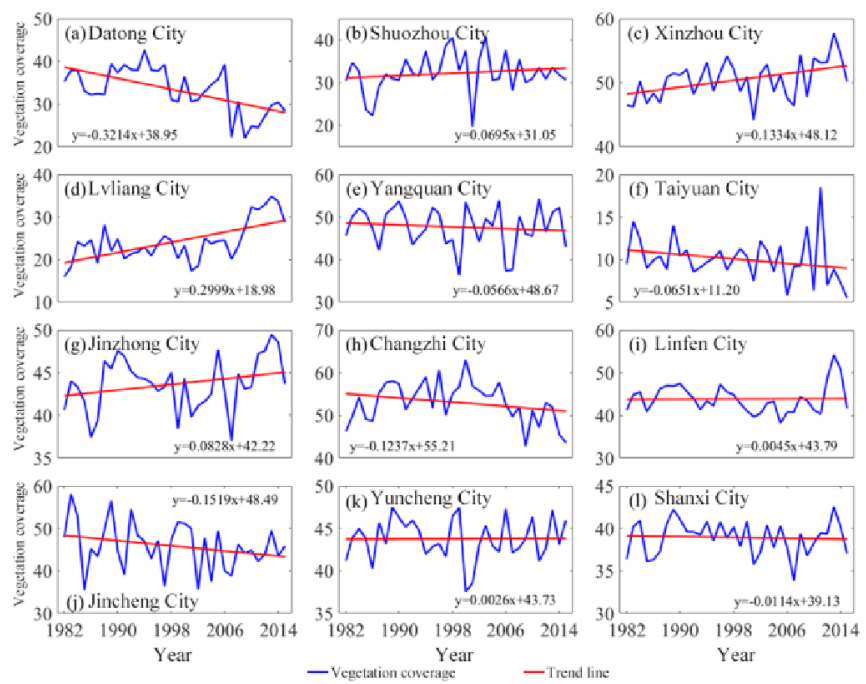

Fig. 6. Interannual change trend of vegetation coverage in Shanxi Province. 


\subsubsection{Assessment of ecological vulnerability}

Figure 7 shows the spatial distribution of NDVI and NPP vulnerability index of climat Fig. e change after K-means cluster analysis. From the spatial distribution of NDVI vulnerability, it can be seen that the extreme vulnerability area is mainly concentrated in the west of Lvliang City, and the ecology of Yizhou City, Yangquan City, Jinzhong City, Changzhi City, Jincheng City and other places is not vulnerable or slightly vulnerable to climate change. The vulnerability of NPP to climate change is roughly the same as that of NDVI, but the extremely vulnerable areas are slightly different.The extremely vulnerable areas of NPP are mainly located in parts of Lvliang City and Linfen City a small part of Yuncheng City. In Changzhi City, Jincheng City, most of the areas show no vulnerability. Table 1 shows the ratio of different vulnerability grades of NDVI and NPP to the total area of Shanxi Province.
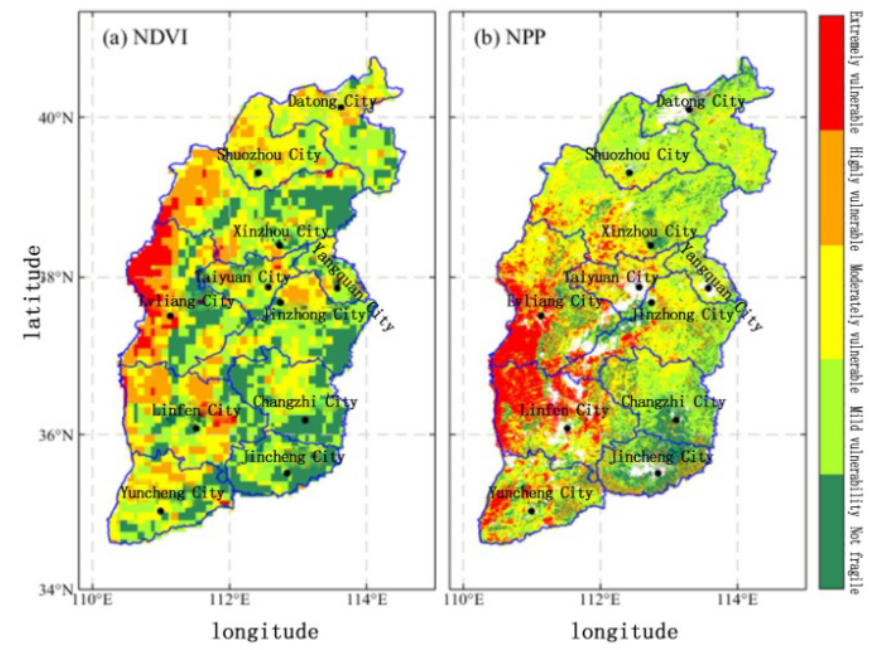

Fig. 7. Vulnerability level of NDVI and NPPV to climate change in Shanxi Province.

Table 1. Ratio of NDVI and NPPV vulnerability to total area of Shanxi Province.

\begin{tabular}{cccccc}
\hline \multirow{2}{*}{ Type } & \multicolumn{5}{c}{ Occupy(\%) } \\
\cline { 2 - 6 } & Not fragile & $\begin{array}{c}\text { Mild } \\
\text { vulnerability }\end{array}$ & $\begin{array}{c}\text { Moderate } \\
\text { vulnerability }\end{array}$ & $\begin{array}{c}\text { Highly } \\
\text { fragile }\end{array}$ & $\begin{array}{c}\text { Extremely } \\
\text { fragile }\end{array}$ \\
\hline NDVI & 22.49 & 29.49 & 28.16 & 15.98 & 3.38 \\
NPP & 14.24 & 31.39 & 34.25 & 14.88 & 5.25 \\
\hline
\end{tabular}

\subsection{Evaluation of comprehensive ecological index}

The calculation of ecological assessment index in this paper is based on ArcGIS platform. Firstly, the ecological assessment factors are normalized, the weights of NPP, NDVI and PWV on ecological environment quality are determined by AHP, and the Spatial Analyst module in ArcGIS software is used to calculate the total ecological assessment index of land reclamation project area. The formula is: $\mathrm{EQ}=\sum_{\mathrm{i}=1}^{4} \mathrm{~s}_{\mathrm{i}} \times \mathrm{t}_{\mathrm{i}}$, EQ is the comprehensive ecological assessment index of the research area, $s_{i}$ is the normalized value of each ecological assessment factor; $t_{i}$ is the proportion of each ecological assessment factor, as shown in Table 2. Figure 8 shows the change trend of the comprehensive ecological assessment index of each city in Shanxi Province in recent years. It can be seen from the figure that the comprehensive ecological assessment index of Shuozhou City, Xinzhou City, 
Lvliang City, Taiyuan City, Jinzhong City, Linfen City and Yuncheng City as well as Shanxi Province as a whole shows an increasing trend, while other cities show a decreasing trend. Among them, Yangquan City, Changzhi City, Jincheng City and Yuncheng City and so on overall ecological assessment comprehensive index level is high, while Taiyuan City ecological assessment comprehensive index level is low.

Table 2. Weight of each evaluation index.

\begin{tabular}{cccccc}
\hline \multicolumn{5}{c}{ Evaluation index 1 } & \multicolumn{4}{c}{ Evaluation index 2 } \\
\hline Target & NDVI & PWV & NDVI & PWV & NPP \\
Weight & $7 / 8$ & $1 / 8$ & $7 / 8$ & $1 / 16$ & $1 / 16$ \\
\hline
\end{tabular}
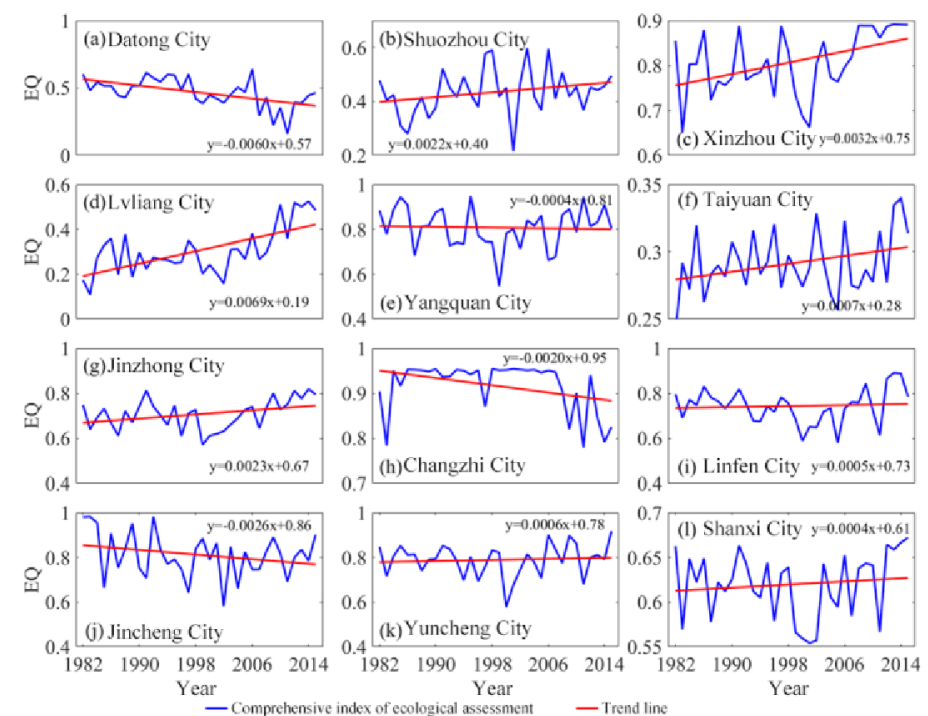

Fig. 8. Change trend of comprehensive index of ecological assessment in Shanxi Province.

\section{Closing remarks}

Based on the technology of GNSS and RS, this paper studies the dynamic monitoring and evaluation methods of ecological restoration in Shanxi mining area, and reflects the dynamic changes of ecological environment by analyzing the spatial and temporal characteristics of NDVI, PWV, NPP, temperature and rainfall. The results are as follows:(1) The PWV variation trend of grid points in Shanxi Province is obvious, and the decreasing rate from north to south gradually becomes larger. As a result of coal mining, the overall vegetation coverage is decreasing. (2) In the construction of multi-factor ecological model, the effect of modeling by machine learning is better than that based on least square regression and the precision of BP neural network is better than that of SVM. (3) According to the statistics of the area variation of typical reservoirs, it is found that the water body area of each reservoir shows a decreasing trend. (4) According to the analysis of the comprehensive ecological assessment index, Shanxi Province shows an overall growth trend, but the growth rate is low. Therefore, the ecological restoration of mining area is still the key issue in the future. 
Thank you for the NPP data provided by the National Aeronautics and Space Administration (National Aeronautics and Space Administration, NASA) and the GIMMS NDVI3g data provided by Clark Laboratories.

\section{References}

1. Sijia Li, Jinman Wang, Zhaotong Zhang.Research Progress in Ecological Compensation for Mineral Resources Development[J].Journal of Ecology, 2019,38 (05): 287-295.

2. Jiaqi Zhang, Yan Wang, Zhengxing Ren, Yonglei Zhao. Exploration on Allocation Mode of Green Ecological Restoration Plants in Mining Area - Taking Da Liu Ta in Shen $\mathrm{Mu}$ City,Shan Xi Province as an example[J]. State Academy of Forestry Administration Journal, 2019,18(03):28-32.

3. Kun Yang. The Coal Mining Subsidence Environmental Effect and Ecological Restoration Techniques-Taking Huainan Coal Mine for Example[D]. Nanjing University of Science and Technology, 2011.

4. Yanke Shen, Linxin Zhang, Xingwei Zhao, et al. Ecological Impact Prediction and Evaluation of an Iron Mine in Xinjiang Based on '3S' Technology[J]. Forest Inventory and Planning, 2017, 42(03): 9-12+72.

5. Bingyan Fan, Junfei Cheng, Xueping Li. Restoration of Ecological Environment in Mining Area[J]. Energy Conservation and Environmental Protection, 2019(02): 42-43.

6. Xiaolei Tian, Baofu Li, Xuewei Li, et al. Spatiotemporal Variation of Precipitable Water and Its Influencing Factors in the North China Plain During 1970-2012[J]. Journal of Liao Cheng University (Nature Science), 2019,32(03):81-88.

7. SAASTAMOINEN J. (1972). Atmospheric correction for the troposphere and stratosphere in radio ranging satellites. The use of artificial satellites for geodesy, 15: 247-251.

8. Huping Hou. Monitoring and evaluation of Net Primary Productivity change in coal mine based on remote sensing[D]. China University of Mining and Technology, 2010.

9. Dongya Cheng, Xudong Li, Jiangzhou Yang. Research on NDVI variation characteristics and precipitation sensitivity of the Yuanjiang River Basin in Guizhou Province[J]. Acta Ecologica Sinica, 2020,40(04):1161-1174.

10. Xiaojun $\mathrm{Fu}$, Linlin Yuan, Xiang Wei. Comparison and analysis of water extraction methods based on remote sensing Image[J]. Geomatics and spatial information technology, 2019,42(08):158-160.

11. RODGERS J L, NICEWANDER W A. Thirteen Ways to Look at the Correlation Coefficient[J]. American Statistician, 1988, 42(1):59-66.

12. Li Wu, Dejiang Liu. Ecological security evaluation of the cultivated land in Yuxi based on GA-BP neural network[J]. Journal of Yunnan Agricultural University (Natural Science), 2019,34(05):874-883.

13. WANG X, ZHONG Y. Statistical learning theory and state of the art in SVM[C]// IEEE International Conference on Cognitive Informatics. IEEE, 2003.

14. Min He, Hesong Wang, Jianxin Sun. Characters of ecosystem vulnerability in southwestern China based on vegetation productivity[J]. Chinese Journal of Applied Ecology,2019, 30(02):429-438. 
15. Li Yu. Assessment on ecosystem vulnerability to drought - The case study of the middle and lower reaches of the Yangze River[J]. Resources and Environment in the Yangze Basin, 2014,23(07):1063-1070.

16. Hongmin $\mathrm{Xu}$. Study on the prediction and assessment methods of water environment quality based on support vector machines theory[D]. Jilin University, 2007.

17. Junguo Wang. Research of modeling methods and control strategies based on neural networks[D]. Huazhong University of Science and Technology, 2004. 